\title{
CRITICAL POINTS OF RATIONAL FUNCTIONS WITH SELF-INVERSIVE POLYNOMIAL FACTORS
}

\section{F. F. BONSALL AND MORRIS MARDEN}

1. Introduction. A polynomial is said to be self-inversive if its zeros are symmetric in the unit circle $C:|z|=1$. Let $E$ be an arbitrary subset of the finite complex plane $Z$, and let $\mathfrak{N}(f, E)$ and $\mathfrak{B}(f, E)$ denote respectively the total multiplicity of the zeros and poles in $E$ of a function $f$. Let $\mathfrak{Q}(f, E)$ denote the number of distinct poles of $f$ in $E$, and let $f^{\prime}$ denote the derivative of $f$. In this notation, Cohn's Theorem ${ }^{1}$ states that, if $f$ is a self-inversive polynomial, then

$$
\mathfrak{N}\left(f^{\prime},|z|>1\right)=\mathfrak{N}(f,|z|>1) .
$$

The theorem of Lucas [3, p. 14] states that if $g$ is any polynomial for which $\mathfrak{N}(g,|z|>1)=0$, then $\mathfrak{N}\left(g^{\prime},|z|>1\right)=0$. A result (Bôcher's Theorem $)^{2}$ due to Walsh states, in effect, that if $\phi$ is a rational function for which $\mathfrak{N}(\phi,|z|>1)=\mathfrak{B}(\phi,|z| \leqq 1)=0$, then

$$
\mathfrak{N}\left(\phi^{\prime},|z| \leqq 1\right)=\mathfrak{N}(\phi,|z| \leqq 1)-1,
$$

provided $\phi=k / K$ with degree $K \leqq$ degree $k$.

These three theorems are special cases of the following, which is our principal result.

THEOREM 1. Let $\phi=k / K$ be a rational function in which the degree of the polynomial $k$ is greater than that of the polynomial $K$. Let $k=f g$ and $K=F G$ where $f, g, F, G$ are polynomials, $f$ and $F$ are self-inversive, and $\mathfrak{N}(g,|z|>1)=\mathfrak{N}(G,|z|<1)=0$. Then

$$
\mathfrak{N}\left(\phi^{\prime},|z|>1\right)=\mathfrak{N}(\phi,|z|>1)+\mathfrak{Q}(\phi,|z| \geqq 1) .
$$

Corollary 1.

$$
\mathfrak{N}\left(\phi^{\prime},|z| \leqq 1\right)=\mathfrak{N}(\phi,|z| \leqq 1)+\mathfrak{Q}(\phi,|z|<1)-1 .
$$

The proof of Theorem 1, given in $\$ 3$, is simple in principle though slightly complicated in detail owing to the possible presence of poles on $C$. Lemma 1 shows that the only zeros of $\phi^{\prime}$ on $C$ are the multiple zeros there of $\phi$. We may therefore vary $\phi$ continuously without

Presented to the Society, December 28, 1951 and December 29, 1952; received by the editors January 28, 1953 and, in revised form, June 29, 1953.

${ }^{1}$ See [2]. A simpler proof of this theorem was given in [1]. Another, [6], was published since the announcement of the present paper.

${ }^{2}$ See [4, pp. 97-99]. This book gives a number of other interesting results on the zeros of self-inversive polynomials, particularly on pp. 52-55, 132-135, 159-163. 
changing $\mathfrak{N}\left(\phi^{\prime},|z|<1\right)-\mathfrak{B}\left(\phi^{\prime},|z|<1\right)$ until we arrive at a rational function for which this number may be counted. If applied to the case when $K \equiv g \equiv 1$, this method would yield a new and simpler proof of Cohn's theorem.

\section{Three lemmas.}

Lemma 1. Under the hypotheses of Theorem $1, \phi^{\prime}(t)=0$ for $t$ on $C$ if and only if $t$ is a multiple zero of $\phi(z)$.

Proof. Without loss of generality, we may assume that $t=1$. If $\phi(1) \neq 0$, then $\phi^{\prime}(1)=0$ would imply

$$
\begin{aligned}
0=\phi^{\prime}(1) / \phi(1) & =\left[f^{\prime}(1) / f(1)\right]+\left[g^{\prime}(1) / g(1)\right] \\
& -\left[F^{\prime}(1) / F(1)\right]-\left[G^{\prime}(1) / G(1)\right] .
\end{aligned}
$$

Let the zeros of $f, g, F, G$, be denoted by $a_{j}, b_{j}, A_{j}, B_{j}$, respectively, and for any complex number $z$ let $z^{*}$ denote $(1-z)^{-1}$. Then $u, v, U, V$, defined by

$$
\begin{array}{rlrl}
m u & =f^{\prime}(1) / f(1)=\sum_{1}^{m} a_{j}^{*}, & M U & =F^{\prime}(1) / F(1)=\sum_{1}^{M} A_{j}{ }^{*}, \\
n v & =g^{\prime}(1) / g(1)=\sum_{1}^{n} b_{j}^{*}, & N V=G^{\prime}(1) / G(1)=\sum_{1}^{N} B_{j}^{*},
\end{array}
$$

where $m, n, M, N$ denote the degrees of $f, g, F, G$, are the centroids of the $a_{j}^{*}, b_{j}^{*}, A_{j}^{*}$, and $B_{j}^{*}$ respectively. Since $w=(1-z)^{-1}$ maps the closed interior of $C$ upon the half-plane $\operatorname{Re}(w) \geqq 1 / 2$ and preserves symmetry in $C$, we have

$$
\begin{gathered}
\operatorname{Re}(u)=\operatorname{Re}(U)=1 / 2, \quad \operatorname{Re}(v) \geqq 1 / 2, \quad \operatorname{Re}(V) \leqq 1 / 2 ; \\
\operatorname{Re}\left[\phi^{\prime}(1) / \phi(1)\right]=\operatorname{Re}[m u+n v-M U-N V] \\
\geqq(m+n-M-N) / 2>0 .
\end{gathered}
$$

As this contradicts (2.1), $\phi^{\prime}(1)=0$ if and only if also $\phi(1)=0$.

The following generalization of Rouché's Theorem may be proved by a method similar to that given in [5, p. 191-192] for the usual Rouché Theorem. It will be convenient to write $(\mathfrak{N}-\mathfrak{B})(f, E)$ in place of $\mathfrak{R}(f, E)-\mathfrak{B}(f, E)$.

Lemma 2. Let $D$ be the interior domain of a simple closed rectifiable curve $\kappa$. Let $f$ and $g$ be regular in a domain containing the closure of $D$ except perhaps for poles in D. If $|g(z)|<|f(z)|$ on $\kappa$, then

$$
(\mathfrak{R}-\mathfrak{B})(f+g, D)=(\mathfrak{R}-\mathfrak{B})(f, D) .
$$


Proof. Let $I(\lambda)=(2 \pi i)^{-1} \int_{x}\left[f^{\prime}(z)+\lambda g^{\prime}(z)\right][f(z)+\lambda g(z)]^{-1} d z$. Then $I(\lambda)$ is a continuous function of $\lambda$ for $0 \leqq \lambda \leqq 1$ and, since it takes only integer values, we conclude that $I(1)=I(0)$.

LemMa 3. With $D$, $\kappa$ as in Lemma 2, let $\psi_{\alpha}(z)$ be a meromorphic function of $z$ in $D$ with no zeros on $\kappa$ for each $\alpha$ in $A: 0 \leqq \alpha \leqq 1$. If $\psi_{\alpha}(z)$ is a continuous function of $(\alpha, z)$ for $z \in \kappa, \alpha \in A$, then

$$
(\mathfrak{N}-\mathfrak{P})\left(\psi_{1}, D\right)=(\mathfrak{N}-\mathfrak{B})\left(\psi_{0}, D\right) .
$$

Proof. Use Lemma 2 and a simple covering argument with respect to $A$.

3. Proof of Theorem 1. If $\phi$ has no zeros and no poles on $C$, set

$$
\phi(z)=\sigma \Pi\left(z-\alpha_{i}\right) \Pi\left(z-\beta_{i}\right) \Pi\left(z-\gamma_{i}\right)^{-1} \Pi\left(z-\delta_{i}\right)^{-1}
$$

where $\left|\alpha_{i}\right|<1,\left|\beta_{i}\right|>1,\left|\gamma_{i}\right|<1,\left|\delta_{i}\right|>1, \sigma=$ const. Let

$$
\phi_{\rho}(z)=\sigma \prod\left(z-\rho \alpha_{i}\right) \Pi\left(\rho z-\beta_{i}\right) \Pi\left(z-\rho \gamma_{i}\right)^{-1} \prod\left(\rho z-\delta_{i}\right)^{-1} .
$$

Lemma 1 shows that $\phi_{\rho}^{\prime}$ has no zeros on $C$ for $0 \leqq \rho \leqq 1$. Since, evidently, $\phi_{\rho}^{\prime}$ is a continuous function of $(z, \rho)$ for $|z|=1,0 \leqq \rho \leqq 1$, we may apply Lemma 3 and conclude that

$$
(\mathfrak{R}-\mathfrak{B})\left(\phi_{1}{ }^{\prime},|z|<1\right)=(\mathfrak{R}-\mathfrak{B})\left(\phi_{0}{ }^{\prime},|z|<1\right) .
$$

However, $\phi_{0}(z)=\sigma z^{t}$, where $t=(\mathfrak{R}-\mathfrak{B})(\phi,|z|<1)$. It follows that

$$
(\mathfrak{N}-\mathfrak{B})\left(\phi^{\prime},|z|<1\right)=(\mathfrak{N}-\mathfrak{B})(\phi,|z|<1)-1,
$$

which yields (1.1) due to the relations

$$
\begin{gathered}
\mathfrak{P}\left(\phi^{\prime}, E\right)=\mathfrak{P}(\phi, E)+\mathfrak{Q}(\phi, E), \\
Z:|z|<\infty, \mathfrak{N}\left(\phi^{\prime}, Z\right)=\mathfrak{N}(\phi, Z)+\mathfrak{Q}(\phi, Z)-1 .
\end{gathered}
$$

If $\phi=0$ or $\infty$ on $C$, we may write $k=h_{1} h_{2}, K=H_{1} H_{2}$, where

$$
\begin{aligned}
\mathfrak{N}\left(h_{1},|z| \neq 1\right) & =\mathfrak{N}\left(H_{1},|z| \neq 1\right)=\mathfrak{N}\left(h_{2},|z|=1\right) \\
& =\mathfrak{N}\left(H_{2},|z|=1\right)=0,
\end{aligned}
$$

and apply (3.1) and Lemma 3 to

$$
\Phi(\rho)=h_{1}(\rho z) h_{2}(z) / H_{1}(z / \rho) H_{2}(z), \quad \rho>1 .
$$

We may complete the proof by choosing $\rho$ sufficiently near 1 .

Corollary 2. With $\phi$ defined as in Theorem 1 , let $\psi=1 / \phi$. Then, $\mathfrak{N}\left(\psi^{\prime},|z|>1\right)=\mathfrak{R}(\psi,|z|>1)+\mathfrak{Q}(1 / \psi,|z|=1)+\mathfrak{Q}(\psi,|z|>1)$.

CoROllary 3. $\mathfrak{N}\left(\phi^{\prime},|z|>1\right)$ and $\mathfrak{N}\left(\psi^{\prime},|z|>1\right)$ are left unaltered if $\phi$ is multiplied by a polynomial all of whose zeros are within $C$. 


\section{REFERENCES}

1. F. F. Bonsall and Morris Marden, Zeros of self-inversive polynomials, Proc. Amer. Math. Soc. vol. 3 (1952) pp. 471-475.

2. A. Cohn, Ueber die Anzahl der Wurzeln einer algebraischen Gleichung in einem Kreise, Math. Zeit. vol. 14 (1922) pp. 110-148.

3. M. Marden, The geometry of the zeros of a polynomial in a complex variable, Mathematical Surveys, vol. 3, American Mathematical Society, 1949.

4. J. L. Walsh, Location of the critical points, Amer. Math. Soc. Colloquium Publications, vol. 34, 1950.

5. L. Bieberbach, Lehrbuch der Funktionentheorie, vol. 1, Leipzig 1930.

6. G. Ancochea, Zeros of self-inversive polynomials, Proc. Amer. Math. Soc. vol. 4 (1953) pp. 900-902.

UNIVERSity OF DURHAM, NEWCASTLE-ON-TyNe, AND University OF Wisconsin, MilwaUkeE 